\title{
A Comparitive Study on Negative Pressure Dressing Versus Conventional Dressing in the Management of Diabetic Foot Ulcer
}

Sri Valli Vemulapalli", Dr. Shivananda, Dr. Balasubramanya K.S, Dr. Tej Swaroop Pala

Department of General Surgery, PESIMSR, College in Kuppam, Andhra Pradesh

Article History

Received: 20.09.2020

Accepted: 09.10.2020

Published: 17.10 .2020

Journal homepage:

https://www.easpublisher.com/easjms

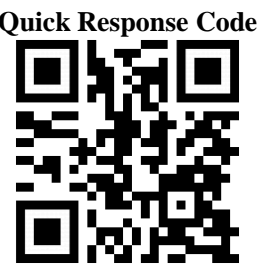

Abstract: Introduction: Diabetic foot ulcers constitute one of the most important complications of diabetes mellitus, with a staggering $25 \%$ lifetime risk. If not treated promptly, progression of infection and sepsis may necessitate a limb amputation. Studies from western population have shown significant implication of vacuum-assisted closure (VAC) therapy in various wounds including Diabetic foot ulcers. Considering the early onset of complications including diabetic foot ulcers in Indian diabetic patients due to differences in lifestyle, culture, socioeconomic status and health education, the role of VAC therapy needs to be studied to establish the efficacy and safety of VAC therapy in the management of diabetic foot ulcers in an Indian population. Materials and methods: Randomized controlled trail conducted in the Department of General surgery PESIMSR from January 2018 to December 2018, in patients with Grade 1 and 2 diabetic foot ulcers (As defined by Wagner's classification). Results: Granulation tissue appeared in 28 patients by the end of week-2 in group I, while it appeared in 11 patients by that time in Group II. Wound was favourable for grafting in Group I compared to Group II by 4 weeks. Conclusion: In the present study it was concluded that the rate of granulation tissue formation, overall graft survival and patient compliance was better in negative pressure dressing group compared to conventional dressing group.

Keywords: Comparitive Pressure Dressing Diabetic Foot.

Copyright (C) 2020 The Author(s): This is an open-access article distributed under the terms of the Creative Commons Attribution 4.0 International License (CC BY-NC 4.0) which permits unrestricted use, distribution, and reproduction in any medium for non-commercial use provided the original author and source are credited.

\section{INTRODUCTION}

Diabetes is a disease characterized by the 'too great emptying of urine' finds its place in antiquity through Egyptian manuscripts dating back to 1500 B.C[1]. Indian physicians called it madhumeha ('honey urine') because it attracted ants. The ancient Indian physician, Sushruta, and the surgeon Charaka (400-500 A.D.) were able to identify the two types, later to be named Type I and Type II diabetes [2].

In the modern era, lifestyle is playing a major role in the development of diseases, particularly noncommunicable diseases like Diabetes Mellitus, Hypertension, Coronary artery diseases, cancer, and other NCDs. The prevalence of diabetes rose globally from $4.7 \%$ in 1980 to $8.5 \%$ in 2014 among adults over 18 years of age [3].

Even with the advances in technology, the complications due to diabetes are causing a major burden in terms of economy. One of the challenges is the foot ulcers, which are of a chronic non-healing type. The peculiarity of the chronic ulcers is that they refuse to heal, and many methods are being developed for their management.
There is no specific ideal treatment for the management of wounds. The standard treatment for diabetic foot ulcers is wound debridement, management of infection, and doing revascularization procedures whenever needed, and off-loading the ulcer [4].

Other methods like usage of hyperbaric oxygen, advanced wound care products, and negative pressure wound therapy will act like add on-therapy [5]. But there is no strong evidence as far as how far these add-on therapies are useful and cost-effective.

This study is being carried out to know the efficacy of the negative pressure wound therapy over conventional therapy.

Controlled application of negative pressure wound therapy has got an important role in assisting wound healing. Negative Pressure Wound Therapy is an invaluable tool and involves the application of subatmospheric pressure to a wound using a computerized unit to covey negative pressure intermittently or continuously to promote wound healing [6]. Negative pressure wound therapy (NPWT) is a useful adjunct in the management of chronic and complex wounds to promote healing and wound bed preparation for surgical procedures such as skin grafts and flap surgery [7]. 
NPWT has been advocated as a novel therapy in the healing of diabetic foot ulcers by stimulating the chronic wound environment in such a way that it reduces the bacterial burden and chronic interstitial wound fluid, increases vascularity and cytokine expression and to an extent mechanically exploiting the viscoelasticity of peri-wound tissues [8].

Vacuum-assisted closure is extremely effective in removing exudate and reducing edema while leaving the surface of the wound moist [9]. NPWT has the potential to benefit a large number of patients from both a symptom and management of wound healing perspective. NPWT provides a splinting effect (rigid support) [10].

Negative Pressure Wound Therapy (NPWT)is an noninvasive, active wound management system, which uses negative pressure in order to enhance the healing process and is also known as Vacuum Therapy (VT), Vacuum-Assisted Closure (VAC), Topical Negative Pressure Therapy (TNPT) or Vacuum Sealing (VS) [11]. Thus the present study is conducted to see the effectiveness of Negative Pressure Wound Therapy (NPWT) over the Conventional method of wound dressing in Diabetic foot ulcers.

\section{Methods And Methodology}

Present study is a randomisied control interventional study conducted in patients admitted in PESIMSR with Diabetic foot ulcer (Grade 1 \& 2) between January 2018-June 2019

\section{Study Population}

All patients fulfilling the inclusion criteria admitted in the Department of general surgery.

\section{Inclusion criteria}

- $\quad$ Patients 20-70 years of age.

- Patients with grade 2 or 3 Diabetic foot ulcer (as defined by Wagner's classification)

\section{Exclusion criteria}

- Patients who are seriously ill.

- Osteomyelitis of underlying bone.

- People on medications, such as corticosteroids and immunosuppressive agents.

- Pregnant or nursing mothers.

\section{Sample size}

- The sample size was calculated using the formula

$$
\mathrm{n}=(\mathrm{Z} \alpha / 2+\mathrm{Z} \beta) 2 *(\mathrm{p} 1(1-\mathrm{p} 1)+\mathrm{p} 2(1-\mathrm{p} 2)) /(\mathrm{p} 1-\mathrm{p} 2) 2
$$

With P1: $66 \%$ P2: $28 \%$ with a power of $80 \%$ and alpha error at $5 \%$ [7]. Based on the study conducted by Lone et al. considering the disappearance of discharge from the wound as a proxy indicator for healing using the above formula, a Sample size came to be 52 with 26 subjects in each group. In the present study, a total of 60 subjects were included.

\section{Sampling technique}

Consecutive Sampling technique was adopted while recruiting and participants were divided into groups by using block randomization to ensure equal distribution of participants in both the groups.

\section{Methods of Collection of DATA}

Subjects admitted into Surgical Department who fulfill the inclusion criteria and willing to enroll and with similar baseline characteristics (such as ulcer location, size, HbA1c values, etc.) were taken into the study.

Using a structured proforma detailed clinical examination and relevant investigations of all the subjects were done. Wounds were thoroughly debrided. Ulcer dimension, as well as surface area, was observed before dressing for all the subjects. Both group subjects were initially started on the same Antibiotic and based on culture, and sensitivity reports Antibiotic has changed accordingly.

The conventional group was subjected to daily dressing by Conventional methods, whereas the test group was subjected to Negative pressure dressing and were left undisturbed till the wound granulates.

Using block randomization, the study subjects were allocated to Negative-pressure dressing (Group A) or Conventional Dressing group (Group B).

The wound for both groups will be observed every five days.

Measurements: Rate of granulation tissue formation. A comparison was made based on the following parameters.

1. Rate of granulation tissue formation

2. Present dimension and surface area of ulcer.

\section{Tools \& techniques used}

Technique for Negative Pressure Wound Technique is as follows: "protect the peri-wound by applying a skin barrier then it should be followed by a transparent film." a dressing or filler material is fitted to the contours of a wound (which is covered with a nonadherent dressing film), and the overlying foam is then sealed with a transparent film. A drainage tube is connected to the dressing through an opening of the transparent film. A vacuum tube is connected through an opening in the film drape to a canister on the side of a vacuum pump or vacuum source, turning an open wound into a controlled, closed wound while removing excess fluid from the wound bed to enhance circulation and remove wound fluids. This creates a moist healing 
environment and reduces edema. "There must be an airtight seal for this therapy to be successful."

Three types of filler material are used over the wound surface: open-cell foam, gauze, and transparent film, or honeycombed textiles with a dimpled wound contact surface. Foam dressings are used to fill open cavity wounds and can be cut to size to fit wounds. The foam dressing is applied, filling the wound, and then a film drape is applied over the top to create a seal around the dressing.

Open weave cotton gauze can be covered with a transparent film, and a flat drain is sandwiched in gauze and placed onto the wound. The film drape covers the wound and creates a complete seal, and then the drain is connected to the pump via the tubing.

Layers of non-woven polyester, joined by a silicone elastomer, has a non-adherent wound contact surface made up of numerous small semi-rigid dome structures.

With all three techniques, once the dressing is sealed, the vacuum pump can be set to deliver continuous or intermittent pressures, with levels of pressure depending on the device used, varying between -125 and $-75 \mathrm{mmHg}$ depending on the material used and patient tolerance. Pressure can be applied constantly or intermittently.

\section{AnAlysis OF DATA}

The Data was entered into MS EXCEL 2016 version and Analyzed. The descriptive data were analyzed as follows: Categorical data were analyzed using percentages, and the continuous data were analyzed using mean and standard deviation. For inferential statistics, Chi-square test and T-test were used.

\section{RESULTS}

In the present study, a total of 60 patients with grade 2 or 3 Diabetic foot ulcers included. These are divided into two groups: the Control group included 30 participants who received the regular type of dressing, and the test group included 30 participants who received negative pressure dressing.

- The mean age of the Control group is 54.2 \pm 11.75 , and the mean age of the Test group is 55.8 \pm 11.04 . The mean ages in both groups were similar, and the difference between both groups is not statistically significant.

- There is an equal gender distribution in both Control and test groups.

- The mean duration of stay in Test group $27.23 \pm$ 6.73 when compared to the Control group where the mean duration is $57.47 \pm 11.933$. The mean duration of stay in the Test group is significantly less when compared to the Control group stating that negative pressure dressing leads to faster healing than conservative techniques.

\begin{tabular}{|l|l|l|l|l|l|}
\hline & GROUP & N & Mean & Std Deviation & P-Value \\
\hline \multirow{2}{*}{$\begin{array}{l}\text { DURATION } \\
\text { OF STY }\end{array}$} & CONTROL & 30 & 57.47 & 11.933 & 0.001 \\
\cline { 2 - 6 } & TEST & 30 & 27.23 & 6.73 & SIG \\
\cline { 2 - 6 } & & & & & \\
\hline
\end{tabular}

- In the control group, $73 \%$ of the participants had a successful outcome, and $26 \%$ had delayed healing. In the test group, $86 \%$ had Success. Out of 4 delayed, 3 were males and one female patient. The total test group had an early and successful outcome.

\begin{tabular}{|l|l|l|l|}
\hline GROUP & \multicolumn{3}{|c|}{ OUTCOME } \\
\hline & & FREQUENCY & PERCENT \\
\hline \multirow{3}{*}{ CONTROL } & SUCCESS & 22 & 73.3 \\
\cline { 2 - 4 } & DELAYED & 8 & 26.7 \\
\hline \multirow{2}{*}{ TEST } & SUCCESS & 26 & 86.7 \\
\cline { 2 - 4 } & DELAYED & 4 & 13.3 \\
\hline
\end{tabular}

- The mean duration of Granulation in Test group $94.35 \pm 3.97$ when compared to the Control group where the mean duration is $60.9 \pm 16.08$. The mean duration of Granulation in the Test group is significantly more when compared to the Control group stating that negative pressure dressing producing better granulation than conservative techniques.
- The mean of Graft Uptake in the Test group is $86 \pm$ 17.88 when compared to the Control group, where the mean is $68.53 \pm 13.367$. The mean of Graft Uptake in the Test group is significantly more when compared to the Control group that negative pressure dressing has excellent graft uptake than conservative techniques. 


\begin{tabular}{|l|l|l|l|l|l|}
\hline \multirow{2}{*}{ GRAFT } & Group & N & Mean & Std Deviation & P Value \\
\cline { 2 - 6 } & CONTROL & 30 & 68.53 & 13.367 & 0.001 \\
\cline { 2 - 6 } & TEST & 26 & 86 & 17.885 & SIG \\
\hline
\end{tabular}

\section{LIMITATIONS}

1. Our study was institution based; multicentric trials can provide better results.

2. The power of the study can be increased by increasing the sample size.

3. On the contrary to conventional therapy, negative pressure therapy cannot be used when the resources are limited, like in the case of Primary health care settings.

\section{CONCLUSION}

The present study emphasizes that the appearance of granulation tissue and graft uptake is well observed in the negative pressure dressing group (test) when compared to the conventional method group(control).

For the treatment of Diabetic foot ulcers, applying a negative pressure treatment technique is the better procedure when compared to the conservative techniques for better healing and early discharge.

\section{REFERENCES}

1. Lakhtakia, R. (2013). The history of diabetes mellitus. Sultan Qaboos University Medical Journal, 13(3), 368.

2. Tipton, C. M. (2008). Susruta of India, an unrecognized contributor to the history of exercise physiology. Journal of applied physiology.

3. Emerging Risk Factors Collaboration. (2010). Diabetes mellitus, fasting blood glucose concentration, and risk of vascular disease: a collaborative meta-analysis of 102 prospective studies. The Lancet, 375(9733), 2215-2222.

4. Alexiadou, K., \& Doupis, J. (2012). Management of diabetic foot ulcers. Diabetes Therapy, 3(1), 4.
5. Hinchliffe, R. J., Valk, G. D., JEFFCOATE, W., APELQVIST, J., \& ARMSTRONG, D. (2008). Specific guidelines on wound and wound-bed management. Diabetes/metabolism research and reviews (Print), 24.

6. Bhattacharya, S., \& Mishra, R. K. (2015). Pressure ulcers: current understanding and newer modalities of treatment. Indian journal of plastic surgery: official publication of the Association of Plastic Surgeons of India, 48(1), 4.

7. Lone, A. M., Zaroo, M. I., Laway, B. A., Pala, N. A., Bashir, S. A., \& Rasool, A. (2014). Vacuumassisted closure versus conventional dressings in the management of diabetic foot ulcers: a prospective case-control study. Diabetic foot \& ankle, 5(1), 23345.

8. Amin, N., \& Doupis, J. (2016). Diabetic foot disease: from the evaluation of the "foot at risk" to the novel diabetic ulcer treatment modalities. World journal of diabetes, 7(7), 153.

9. Kavitha, K. V., Tiwari, S., Purandare, V. B., Khedkar, S., Bhosale, S. S., \& Unnikrishnan, A. G. (2014). Choice of wound care in diabetic foot ulcer: a practical approach. World journal of diabetes, 5(4), 546.

10. Othman, D. (2012). Negative pressure wound therapy literature review of efficacy, cost effectiveness, and impact on patients' quality of life in chronic wound management and its implementation in the United kingdom. Plastic surgery international, 2012.

11. Mafi, R., Malahias, M., Jordan, D. J., \& Hindocha, S. (2014). The evidence-based principles of negative pressure wound therapy (NPWT) dressing applications; A review of the literature. Int J Infect Control, 10, i2 